In a comparison of multiple solid tumour types in mice, antiPD1-responsive tumours had markedly higher levels of baseline CXCL9 and CXCL10 expression than non-responsive tumours, which suggests that the expression of CXCR3 ligands might have predictive potential. Indeed, in plasma samples from patients with melanoma who had received anti-PD1 therapy, the levels of CXCR3 ligands early after therapy correlated with treatment outcome. Furthermore, the authors showed that a non-responsive tumour type could be made responsive to anti-PD1 therapy by upregulating DC expression of CXCL9 using epigenetic modulators. Thus, the CXCR3 system might have future potential both as a biomarker of treatment success and as a target for combination therapy with anti-PD1.

Kirsty Minton

ORIGINAL ARTICLE Chow, M. T. et al. Intratumoral activity of the CXCR3 chemokine system is required for the efficacy of anti-PD-1 therapy. Immunity https://doi.org/10.1016/ j.immuni.2019.04.010 (2019)

of infected mice with the drug FTY720 to transiently block egress from lymphoid organs did not affect weight loss, nor did lack of expression of the cytotoxic mediator perforin, suggesting that cachexia did not involve T cell-mediated cytotoxicity in adipose tissue. However, antigenspecific priming was important, as immunodeficient mice repopulated with ovalbumin-specific $\mathrm{CD} 8^{+} \mathrm{T}$ cells were completely protected against virus-induced weight loss.

Together, the data suggest that following LCMV infection, CD8 ${ }^{+}$ $T$ cells activated by specific antigen and type I interferons promote, possibly through an as-yet-unknown endocrine factor, metabolic reprogramming in adipose tissue. Further research is needed to understand whether cachexia serves to temper immune responses to limit immunopathology or whether it helps to release nutrients to fuel the antiviral immune response.

Lucy Bird

ORIGINAL ARTICLE Baazim, H. et al. CD8 ${ }^{+} T$ cells induce cachexia during chronic viral infection. Nat. Immunol. 20, 701-710 (2019)

\title{
Targeting VCAM1 rejuvenates the brain in aged mice
}

Ageing is associated with the deterioration of brain structure and function; the reasons for this are not clear, but neurodegeneration has been linked to increased activation of microglia and neuroinflammation. Tony Wyss-Coray and colleagues now show that the expression of vascular cell adhesion molecule 1 (VCAM1) on brain endothelial cells (BECs) increases with age and that targeting VCAM1 reverses microglial cell activation and cognitive deficits in the brains of aged mice.

Previously, the same authors had found that factors in the plasma or blood from young mice can improve brain function in aged mice, whereas young mice exposed to plasma or blood components from aged mice showed impaired neurogenesis and cognitive function. As the blood-brain barrier $(B B B)$ is crucial for regulating the transport of cells and molecules into the brain, the current study by Yousef et al. examined how blood endothelial cells (BECs) are affected by ageing. Initial transcriptomic analyses indicated that more than 1,000 genes are differentially expressed by BECs from young (3-month-old) and aged (19-month-old) mice. Notably, aged BECs showed a more inflammatory and activated gene expression profile.

The authors used published proteomic data sets to search for plasma proteins with vascular functions that are expressed in healthy older humans and identified eight such factors that were also expressed at the transcript level by aged mouse BECs. They found that increased expression of soluble VCAM1 (sVCAM1) correlated most strongly with increased age. VCAM1 is upregulated on activated endothelium and facilitates the tethering and transmigration of leukocytes into tissues; it is constitutively cleaved by matrix metalloproteinases and shed into the plasma as sVCAM1. Both humans and mice showed a significant increase in plasma sVCAM1 levels with advanced age. Furthermore, the authors found that the increase in sVCAM1 plasma levels in aged mice correlated with increased expression of VCAM1 on cerebral blood vessels.
Only a small percentage of total BECs expressed VCAM1 at the protein level, but using single-cell RNA sequencing analyses of VCAM1-enriched BECs the authors identified three distinct BEC subpopulations: one BEC population was mainly VCAM1- and showed a capillary phenotype; one was VCAM1+, showed a post-capillary venous phenotype and expressed higher levels of pro-inflammatory genes; and the final subpopulation was VCAM $1^{+}$ and expressed arterial markers and genes involved in vascular remodelling. Parabiosis and plasma infusion experiments showed that exposure to blood or plasma from aged mice increased VCAM1 expression by BECs of young mice and also led to reduced neural progenitor cell (NPC) activity and increased microglial cell activation. Strikingly, inducible deletion of VCAM1 in BECs protected young mice from the detrimental effects of aged plasma transfusion on NPCs and microglia. Anti-VCAM1 treatment had a similar protective effect. Moreover, aged mice that lacked VCAM1 expression on BECs or that had been treated with anti-VCAM1 had increased NPC activity and reduced activation of microglia compared with controls. Finally, in behavioural studies, anti-VCAM1 treatment improved the performance of aged mice in tests designed to assess age-related impairments in memory and learning.

The authors did not see any significant differences in BBB permeability or in the numbers of different leukocyte populations in the brains of young or aged mice following VCAM1 deletion from BECs. Therefore, they propose that increased levels of pro-inflammatory mediators in the blood of aged mice may induce the activation of BECs and enhance tethering of leukocytes to brain endothelium, thereby leading to the delivery of inflammatory signals to the brain parenchyma that impair cognition.

Yvonne Bordon

ORIGINAL ARTICLE Yousef, H. et al. Aged blood impairs hippocampal neural precursor activity and activates microglia via brain endothelial cell VCAM1. Nat. Med. https://doi.org/10.1038/ s41591-019-0440-4 (2019) 